\title{
Alternativas para optimizar la productividad y asegurar una mejor calidad del espárrago (Asparagus officinalis var. altilis L.)
}

\author{
Alternatives to optimize productivity and ensure a better \\ quality of asparagus (Asparagus officinalis var. altilis L.)
}

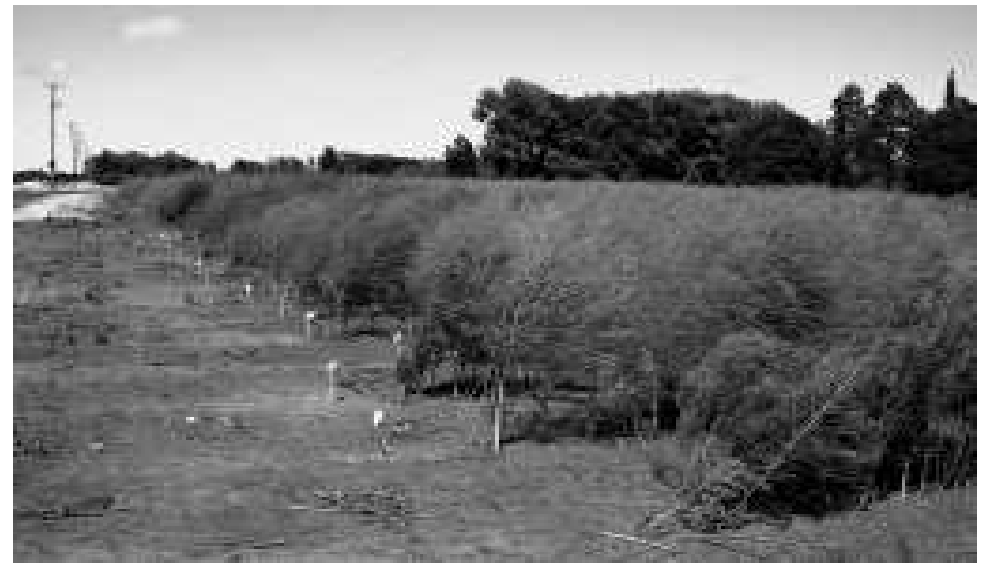

JAVIER ALEJANDRO MARINA

ANA MARÍA CASTAGNINO 2.4

PATRICIA SASTRE V. ${ }^{2}$

KARINA DÍAZ ${ }^{2}$

ANDREA PAOLA GUISOLIS :

Plantación de espárrago

en Azul, Buenos Aires.

Foto: J.A. Marina

\section{RESUMEN}

El espárrago constituye una alternativa productiva innovadora, con potencial para el mercado global; si bien cuenta con una demanda creciente, presenta altas exigencias en calidad y en calibres específicos, según el mercado de destino; actualmente se está expandiendo la demanda de turiones cortos, que pueden ser consumidos íntegramente. Con el objetivo de evaluar el impacto sobre la productividad de alternativas tradicionales e innovadoras de producción, en el primer bienio de la etapa adulta, se realizaron dos ensayos: el primero tuvo lugar en la Chacra Experimental de la Facultad de Agronomía (UNCPBA) en Buenos Aires, con diferentes densidades (D1: 25.000 y D2: 17.857 plantas/ha) y tamaños de la corona, arañas o plantines (grandes, $>200 \mathrm{~g}$, medianas, $100-200 \mathrm{~g}$, y chicas, $<100 \mathrm{~g}$ ); con respecto a los turiones, se evaluó el peso comercial, número, peso promedio y distribución de calibres. En el segundo ensayo se evaluó durante un mes la pérdida de peso de turiones con dos tamaños (22 y $12 \mathrm{~cm}$ ) y tres calibres (grande, mediano y pequeño) diferentes. El mayor tamaño de la corona y la mayor densidad mostraron los mejores rendimientos: 9,6 y 8,8 t ha-1 ${ }^{-1}$, respectivamente. Al evaluar el rendimiento durante dos años, se encontró que la producción fue significativamente mayor en el segundo año, con 9,2 t ha-1. Durante la poscosecha, para los distintos largos y calibres, se encontraron diferencias estadísticas significativas en la pérdida del peso, obteniendo los mayores porcentajes de conservación de las características organolépticas en turiones cortos con calibres gruesos y medianos, y envasados.

${ }^{1,3}$ Facultad de Agronomía, Licenciatura en Tecnología de los Alimentos, Universidad Nacional del Centro de Buenos Aires (UNCPBA), Azul, Buenos Aires (Argentina).

2 Facultad de Agronomía, Centro Regional de Estudio Sistémico de Cadenas Agroalimentarias (CRESCA), Universidad Nacional del Centro de Buenos Aires (UNCPBA), Azul, Buenos Aires (Argentina).

3 Autor para correspondencia.amc@faa.unicen.edu.ar 


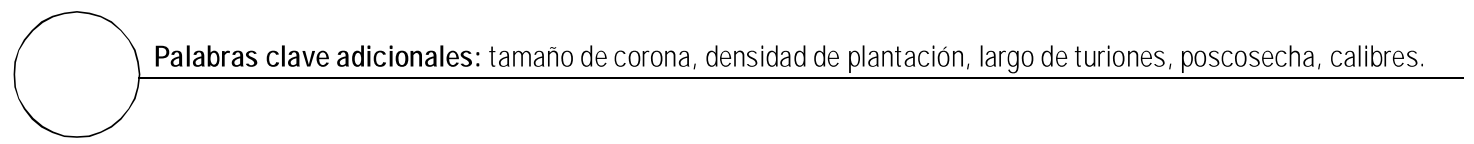

\section{ABSTRACT}

Asparagus is an innovative, productive, alternative plant with a potential for the global market. Although there is an increasing niche market for this crop, there are high demands for quality and specific calibers in accordance with the market. At present, the demand for short turions that can be fully consumed is expanding. In order to evaluate the impact on the productivity of traditional and innovative production alternatives in the first two years of the adult stage, two trials were carried out. The first trial with different densities (D1: 25,000 and D2: 17,857 plants/ha) and crown sizes (large: >200, medium: 100-200, and small: <100 g) took place at the Experimental Unit of Facultad de Agronomía, Universidad Nacional del Centro de Buenos Aires (UNCPBA). Net commercial weight, number of turions, average weight, and distribution of calibers were evaluated. In the second trial, the weight loss of turions of two sizes $(22$ and $12 \mathrm{~cm}$ ) and three different calibers (large, medium and small) was evaluated over a month long period. The highest crown size and the highest density showed better yields of 9.6 and $8.8 \mathrm{t} \mathrm{ha}^{-1}$, respectively. When evaluating the yield for two years, it was found that the production was significantly higher in the second year with $9.2 \mathrm{t} \mathrm{ha}^{-1}$. After harvest, for the different lengths and calibers, there were significant statistical differences found in weight Ioss, with the highest percentages of retained organoleptic characteristics in the short turions with thick and medium calibers, which were packed.

Additional key words: crown size, plant density, turions length, postharvest, calibres

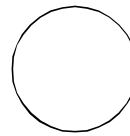

En una alternativa productiva perenne como el espárrago (Asparagus officinalis var. altilis L.) es fundamental determinar su potencial productivo a lo largo de los años, en particular en su etapa adulta, la evolución de su rendimiento en la etapa de cosecha y la calidad del producto obtenido durante la poscosecha. Además, es importante analizar el comportamiento de los turiones según su largo, ya que actualmente se está expandiendo la demanda de turiones cortos, comúnmente llamados puntas de espárragos o tips, cuyo comportamiento aún no ha sido suficientemente estudiado, por su reciente incorporación al mercado.

El espárrago tiene un período promedio de cosecha de 10 años y llega al máximo de producción a los cuatro o cinco años (Ellison, 1986; Asprelli et ál., 2005). En este cultivo se identifican varias etapas productivas: una inicial, de crecimiento exponencial, seguida de otra, de productividad estable, y finalmente una de decrecimiento productivo (Falavigna y Palumbo,
2001); la extensión y productividad de cada una de las etapas dependen de las condiciones de cultivo, como por ejemplo, densidad, tamaño y características del órgano de inicio, manejo del cultivo, extensión del período de cosecha y condiciones agroclimáticas, entre otras.

El espárrago es una especie herbácea plurianual, cuyas plantas están formadas por un tallo principal único, subterráneo y modificado en un rizoma sobre el cual se forman las yemas que dan lugar a los turiones (parte comestible). Al conjunto formado por raíces, tallo principal y yemas se la denomina corona, araña o garra (Benajes-Sanahuja, 1990). Las características de las raíces y yemas, tales como tamaño, calidad y sanidad, tienen un rol decisivo, ya que de ellas depende el rendimiento anual del cultivo, la calidad de la producción y la vida útil de la esparraguera. El tamaño de las yemas que integran las coronas está positivamente correlacionado con el calibre de los turiones producidos (Nichols y Wolley, 1985); por tal 
motivo, es necesario ajustar la densidad de cultivo en correspondencia con los calibres exigidos por el mercado objetivo, ya que los distintos mercados no tienen la misma preferencia de calibres

Es muy importante una correcta elección del órgano de inicio (corona) para lograr un buen resultado en el cultivo (Montanari et ál., 1996), ya que esto define la producción futura y la vida útil de la esparraguera. Numerosos autores han demostrado que errores cometidos durante esa primera etapa repercuten sobre toda la vida del cultivo, en especial cuando se trata de un cultivo iniciado por coronas. Dentro de las ventajas del sistema tradicional de coronas se puede mencionar que si bien demanda un año para su producción, una vez realizado el trasplante, desarrolla un sistema radical muy vigoroso y admite desde el comienzo un manejo de la plantación de secano (Castagnino, 2006). Además, la producción del cultivo mediante este sistema no requiere de tecnologías específicas, como es el caso de las coronas. Los rendimientos que se obtienen son muy variables y están condicionados por la edad de la plantación, la variedad, las condiciones climáticas, el manejo del lote y también el origen de la semilla empleada (Rivera y Rodríguez, 1999).

El cultivo de espárragos ha cobrado gran relevancia; se cuenta en la actualidad con una producción mundial de 800.000 t, con una superficie de 231.500 ha, destacándose en orden de importancia en producción: América (315.000 t), Asia (235.000 t) y Europa (230.000 t), mientras que en superficie cultivada: Asia (93.500 ha), América y Europa (con 65.000 ha cada una), Oceanía (4.750 ha) y África con (3.250 ha). Dentro de Sudamérica se destacan Perú (20.000 ha), Chile (2.700 ha) y Argentina (1.300 ha) (Laemers, 2008).

Para la comercialización de los espárragos, ya sea en el mercado interno o en el externo, se busca obtener un producto de excelente calidad, por tipicidad, seguridad alimentaria y trazabilidad, y sobre una moderna organización comercial. Para mantener el mercado es necesario que los turiones sean verdes en por lo menos $80 \%$ de su largo, turgentes, bien formados y sanos, según el Protocolo de Calidad de Espárrago Fresco (SAGPyA, 2007). Según lo establecido por las normas de comercialización de espárragos (Reglamento 2377/1999), vigente en todos los estados de la Unión Europea a partir del primero de enero de 2000, los espárragos comercializables deben cumplir una serie de requisitos, como: haber sido cosechados en el momento óptimo de crecimiento con las brácteas bien cerradas, estar enteros, sanos, limpios, secos, sin olores ni sabores extraños y con un corte neto en la base.

Luego de ser cosechados, los productos agrícolas continúan sus procesos metabólicos, principalmente la respiración, por lo tanto, se deben controlar los factores que intensifiquen la respiración y, por ende, la maduración. Como consecuencia de la respiración y los procesos metabólicos involucrados en la poscosecha, se pueden reconocer diferentes formas de cambios o pérdidas en los productos agrícolas almacenados: pérdida de agua: el agua es el compuesto más abundante en los productos perecederos (más del 70\% del peso fresco) y es el que más rápido se pierde durante la respiración; pérdida de clorofila y otros pigmentos: es un problema en productos como los tallos (como el espárrago), frutos y hojas, cuyo color intenso y brillante es deseado. Finalmente se pueden dar cambios nutricionales: puede ocurrir pérdida de vitaminas, como la vitamina $\mathrm{C}$, si las condiciones de almacenamiento después de la cosecha no son adecuadas para la mayoría de las frutas. El espárrago posee vitaminas $A, C$ y algunas del complejo B, como tiamina y niacina. Tal como lo encontrado por Manrique (1998), la combinación de todas estas formas de pérdidas puede incidir directamente en una reducción substancial de los atributos de calidad que caracterizan los productos perecibles, y de la vida útil en almacenamiento.

Por ser los principales órganos de transpiración y fotosíntesis de la planta y carecer de capacidad de almacenamiento de fotosintatos, las hojas y tallos son los más susceptibles a un rápido deterioro. Por eso se recomienda su rápida refrigeración, para reducir su temperatura de campo.

El manejo de la temperatura es la técnica disponible más útil e importante para reducir el daño físico y fisiológico en los vegetales frescos luego de la cosecha. Las reacciones metabólicas en hortalizas y frutas se reducen de dos a tres veces por cada $10^{\circ} \mathrm{C}$ de disminución de temperatura (López-Gálvez y Cantwell, 1996); el incremento en los niveles de respiración y 
producción de etileno es minimizado cuando el producto fresco es procesado a baja temperatura, lo que permite detener el deterioro $y$, en particular, inactivar o inhibir las enzimas responsables del pardeamiento; así se disminuye la pérdida de peso, la actividad microbiana y se mitiga el efecto de daños mecánicos, preservando la calidad del producto.

El manejo poscosecha no puede mejorar la calidad del producto cosechado; esto quiere decir que el buen manejo del cultivo es de primordial importancia. El producto mal manejado es de baja calidad y de corta vida útil, lo que impide que alcance mercados exigentes y lejanos; este es un problema que afecta gravemente a la economía de los productores, los comercializadores, los consumidores y, por ende, a todo el país. En los países desarrollados se estima que las pérdidas por poscosecha de los productos hortofrutícolas alcanzan del 5 al 25\%, en tanto que en los países en vías de desarrollo alcanzan del 20 al 50\%, y en algunos casos más.

De las innumerables definiciones que se han dado sobre la calidad se desprende que, por lo general, es el consumidor quien la determina con su aceptación o rechazo. En efecto, él tiene unas necesidades y es necesario ofrecerle productos que las satisfagan. Es, pues, imprescindible asumir que la calidad de un alimento es esencialmente la facultad de dar respuesta a las necesidades de determinados grupos de consumidores, y que, por lo tanto, la producción de frutas y hortalizas frescas (como el espárrago en este caso) deberá adaptarse a este concepto.

Un análisis de la evolución que ha experimentado la sociedad de consumo permite afirmar que los consumidores exigen alimentos más frescos, sanos, puros, nutritivos y con mejores características sensoriales, y piden mayor información sobre su composición y cualidades (Romojaro et ál., 1996); por tal motivo, es evidente que la planificación de la producción, la investigación y la experimentación se deben realizar en función de este concepto. De acuerdo con el perfil del consumidor de la Unión Europea, la calidad sensorial determina que el alimento sea o no consumido, esto comprende aspectos gustativos (dulzor, acidez y amargor), olfativos (aroma, perfume), táctiles (dureza, harinosidad) y su determinación es todavía muy subjetiva (Romojaro et ál., 1996).
Además de las exigencias de calidad, que cada vez son mayores, los consumidores establecen preferencias de acuerdo con el calibre de los espárragos; en los países de Europa optan por los turiones de mayor calibre $(L, X L$ y J) y en Estados Unidos eligen turiones de menor cal ibre (S y M): por este motivo resulta necesario estudiar cómo inciden los distintos marcos de plantación en la productividad alcanzada, no solo en los primeros años del cultivo, cuando se obtiene un crecimiento exponencial, sino fundamentalmente en la etapa adulta.

En el mercado interno de Argentina, los atados de espárrago verde se comercializan en cajones «torito» que tienen un peso neto de $9 \mathrm{~kg}$, con 18 atados de $500 \mathrm{~g}$ (Rivera y Rodríguez, 1999). También se utilizan cajas de cartón plástico de 6 $\mathrm{kg}$ con 12 atados en su interior.

Para la exportación, los espárragos pueden presentarse en los envases colocados en hileras o en manojos firmemente atados, sujetos por cintas o gomas elásticas, o envasados en pequeñas bolsas de polietileno. Dentro de los envases se ordenan en hileras regulares protegidos con papel. Los envases que normalmente se utilizan son las cajas de cartón, con dimensiones de $26 \times 50 \times 24$ $\mathrm{cm}$ (largo $x$ ancho $x$ alto), provistas de tapa, 0 bien se pueden utilizar envases de cartón de una capacidad de $11 \mathrm{~kg}$ con atados de 0,7 kg cada uno. Para cumplir con los parámetros de calidad, Ios turiones no deben sobresalir del nivel superior del envase, deben embalarse de tal manera que queden debidamente protegidos. El material usado para empaque debe ser nuevo (en caso del cartón), estar limpio y ser de calidad tal que evite daños al producto. Se permite el uso de materiales, en particular papel o sellos, que lleven las especificaciones comerciales, siempre y cuando estén impresos o etiquetados con tinta o pegamentos no tóxicos (IICA, 2007). No se encuentra información sobre estudios realizados respecto a las presentaciones de turiones cortos, seguramente porque se trata de una técnica de reciente incorporación en algunos mercados (como es el caso de Perú), por lo cual es importante evaluar su comportamiento.

El objetivo de este trabajo fue evaluar el impacto sobre la productividad en el primer bienio de la etapa adulta de alternativas tradicionales e innovadoras de producción aplicadas a la cadena agroalimentaria del espárrago, a fin de contribuir a incrementar su productividad comercial y a la 
optimización de la calidad de los turiones cosechados.

La significativa disminución de la superficie cultivada a nivel nacional, y las condiciones socioeconómicas actuales de muchos países latinoamericanos que disponen de mano de obra para las producciones intensivas, sumado a la creciente demanda del mercado global, tornan viable alternativas productivas como el espárrago, que para su éxito requiere aplicar el enfoque de cadena, es decir, del cuidado armónico de todos sus eslabones. Estos aspectos hacen que las instituciones, como la Universidad Nacional del Centro de Buenos Aires (UNCPBA), promuevan la realización de proyectos de investigación, y de tesis de grado en el marco de estos, en función del rol que las instituciones deben cumplir.

\section{MATERIALES Y MÉTODOS}

El experimento se llevó a cabo en la Chacra Experimental de la Facultad de Agronomía, de la Universidad Nacional del Centro de la Provincia de Buenos Aires ( $36^{\circ} 48^{\prime}$ S y $59^{\circ} 51^{\prime} \mathrm{W}$ ), sobre Ruta Nacional N. ${ }^{\circ}$, en la zona centro de la Provincia de Buenos Aires. La siembra del almácigo se efectuó manualmente a comienzo de primavera (21 de septiembre de 2001), a una profundidad de $2 \mathrm{~cm}$ y con un marco de siembra de $1,40 \mathrm{~m}$ entre hileras y $0,10 \mathrm{~m}$ entre plantas.

El híbrido utilizado fue el UC 157, heterocigota, muy precoz, con turiones de calibre medio, con brácteas cerradas, aún en condiciones de cosecha con altas temperaturas (Falavigna, 2006). Las coronas, una vez extraídas en forma semimecanizada, el 10 de septiembre de 2003 (efectuando el descalzado mecánico y la extracción manual), fueron lavadas, seleccionadas en chicas, medianas y grandes, y desinfectadas mediante el empleo de dos fungicidas: uno sistémico (Benomil) y uno de contacto (Captan).

La preparación del lote consistió en dos cinceladas cruzadas, dos pasadas de rastra de discos y una de motocultivador. Previo al trasplante se abrieron surcos a una profundidad de $25 \mathrm{~cm}$ con motocultivador equipado con aporcador. Se efectuó fertilización de fondo en bandas de 0,30 m con fosfato diamónico; la dosis utilizada fue de $300 \mathrm{~kg} \mathrm{ha}^{-1}$.
La etapa de plantación a campo se realizó en primavera (12 de septiembre), para lo cual se utilizó un diseño completamente al azar. El número de surcos por parcela fue de tres, y el total, de dieciocho. El largo de los surcos fue de 7 $\mathrm{m}$, por lo que con los tres bloques de evaluación y los caminos de $5 \mathrm{~m}$, el largo del ensayo resultó de $48 \mathrm{~m}$, y el ancho real fue de $28 \mathrm{~m}$, y, con los laterales, el ancho total fue de $36 \mathrm{~m}$, resultando la superficie total en evaluación: $1.728 \mathrm{~m}^{2}$. La plantación se llevó a cabo a los dos años de la siembra, debido a las condiciones climáticas desfavorables del año de siembra (exceso de precipitaciones), que generaron corrimiento de semillas, lo que dificultó la emergencia y la etapa de crecimiento inicial; por tal motivo, se consideró oportuno dar más tiempo a las coronas para que adquieran las características necesarias para el presente ensayo.

Se utilizaron dos densidades de siembra de las coronas, D1: 25.000 plantas/ha y D2: 17.857 plantas/ha; correspondiente a los siguientes marcos de plantación: $1,60 \times 0,25$ m y 1,60 × 0,35 $m$, respectivamente. La profundidad de plantación fue de 0,30 m. Se sembraron coronas de diferentes tamaños, grandes $(>200 \mathrm{~g})$, medianas $(100-200 \mathrm{~g})$ y chicas $(<100 \mathrm{~g})$.

En el bienio en estudio se realizaron las cosechas desde el primero de octubre al 30 de noviembre de 2008, para el primer año de estudio, y desde el 18 de septiembre al 30 de octubre de 2009, para el segundo año. Las cosechas se efectuaban con una frecuencia de día por medio, acompañando la evolución de la temperatura y precipitaciones. Luego de cosechados, los espárragos fueron acondicionados (lavados, cortados y calibrados) y se determinó el número de turiones y su peso.

Con parte de la producción obtenida de dicho ensayo, se efectuó una evaluación de la pérdida de peso fresco de turiones de espárrago verde cortados a diferentes largos: (a) $22 \mathrm{~cm}$ y (b) 12 $\mathrm{cm}$, durante la poscosecha. Luego de cosechados, los espárragos fueron acondicionados según el protocolo de calidad para espárrago fresco (SAGPyA, 2007) y se conservaron a $4 \pm 1{ }^{\circ} \mathrm{C}$ durante un mes, evaluando el peso fresco con una frecuencia de día por medio.

Las variables estudiadas fueron:

(1) Peso comercial neto en $\mathrm{kg}$ por cosecha, obtenido a lo largo del período de la recolección. 
(2) Número de turiones producidos por cosecha.

(3) Peso promedio por turión, en g.

(4) Distribución de calibres, acorde con el protocolo de calidad para espárrago verde de Argentina, agrupados en categorías, según las posibilidades de mercados de destino: grandes (extra large y large) y medianos (medium) para la exportación, y chicos (small y asparagina) para el mercado interno.

(5) Pérdida de peso fresco durante la conservación según el largo de los turiones.

Los datos fueron analizados mediante un análisis Anova y prueba de LSD al $\mathrm{P}<0,05$.

\section{RESULTADOS Y DISCUSIÓN}

\section{Evaluación de la productividad neta comercial de dos años de una plantación adulta tradicional de espárrago verde}

El estudio realizado indica que existiría una relación directamente proporcional entre el rendimiento logrado y la densidad y el tamaño de corona, durante la etapa adulta de una plantación tradicional de espárrago verde, debido a que el análisis efectuado resultó significativo para todos los efectos principales.

\section{Peso fresco del producto cosechado}

La productividad lograda por la plantación adulta en estudio tuvo un rendimiento promedio del bienio de $8.324,43 \mathrm{~kg} \mathrm{ha}^{-1}$ (tabla 1), que resultó alentador, ya que duplica a la media nacional, que es de $3,95 \mathrm{t} \mathrm{ha}^{-1}$.

Como lo encontrado por Falavigna (2004), el rendimiento de la esparraguera, a partir del tercer año desde la plantación (etapa adulta), en cada cosecha la cantidad de turiones obtenidos varió entre 370 y $510 \mathrm{~kg} \mathrm{ha}^{-1}$, en función de la temperatura. Por lo tanto, la plantación produjo en promedio $440 \mathrm{~kg}$ por cosecha, y por planta, la densidad 1 produjo $352 \mathrm{~g}$, y la densidad 2, $437 \mathrm{~g}$. Como muestra la tabla 1, la productividad neta comercial del cultivo durante el bienio evaluado, correspondiente a la cuarta y quinta evaluación, continuó creciendo, ya que se produjo un incremento de $1,7 \mathrm{t}$, lo que indica que pese a haber transcurrido siete años desde la plantación del cultivo, aún no ha llegado a su estabilidad productiva.
Tabla 1. Peso fresco de espárrago obtenido según año, densidad de plantación y tamaño de corona.

\begin{tabular}{|l|c|c|c|}
\hline \multicolumn{1}{|c|}{ Variable } & & $\mathrm{t} /$ cosecha & $\mathrm{t} \mathrm{ha}^{-1}$ \\
\hline \multirow{2}{*}{ Año del bienio en estudio } & 1 & $0,37 \mathrm{~b}$ & $7,5 \mathrm{~b}$ \\
\cline { 2 - 4 } & 2 & $0,51 \mathrm{a}$ & $9,2 \mathrm{a}$ \\
\hline \multirow{2}{*}{ Densidad } & $25.000 \mathrm{pl} / / \mathrm{ha}$ & $0,47 \mathrm{a}$ & $8,8 \mathrm{a}$ \\
\cline { 2 - 4 } & $17.857 \mathrm{pl} . / \mathrm{ha}$ & $0,41 \mathrm{~b}$ & $7,8 \mathrm{~b}$ \\
\hline \multirow{3}{*}{ Tamaño de corona } & Chico & $0,38 \mathrm{~b}$ & $7,6 \mathrm{~b}$ \\
\cline { 2 - 4 } & Mediano & $0,45 \mathrm{a}$ & $9,0 \mathrm{a}$ \\
\cline { 2 - 4 } & Grande & $0,48 \mathrm{a}$ & $9,6 \mathrm{a}$ \\
\hline
\end{tabular}

Los promedios en cada variable con letras distintas indican diferencia significativa según la prueba de LSD $(P<0,05)$.

En la figura 1 se observa la evolución productiva promedio, a lo largo del periodo de cosecha para los dos años considerados, mostrando la diferencia productiva entre ambos. La máxima productividad en peso fresco neto en promedio para ambos años se obtuvo en la cosecha 13 (917 $\mathrm{kg} \mathrm{ha}^{-1}$ ), seguida de las 17 y 10 (con alrededor de $792 \mathrm{~kg} \mathrm{ha}^{-1}$ por cosecha), resultando la diferencia de peso cosechado significativa entre un año y otro. Las cosechas menores fueron de la 1 a la 5 , con aproximadamente $140 \mathrm{~kg} \mathrm{ha}^{-1}$.

A partir de la utilización de dos densidades distintas, dadas no por la distribución espacial de las hileras, que fue similar, sino por la distancia entre plantas, como oportunamente se mencionara, se logró una diferencia significativa entre ellas en el peso fresco comercial obtenido, ya que la mayor densidad permitió obtener mayor productividad que la menor, con una diferencia de 1 t entre ellas (tabla 1), siendo 0,412 $\mathrm{kg} /$ planta la productividad individual promedio para la alta densidad, y 0,365 kg/planta para la baja.

Teniendo en cuenta el valor que posee este producto en el mercado, es muy interesante para los productores saber cómo se comporta la producción con la utilización de diferentes densidades, a la hora de elegir el marco de plantación que van a emplear; ya que en este caso, por ejemplo, la diferencia de $50 \mathrm{~kg}$ en cada cosecha representa la posibilidad de poder comercializar nueve cajas de exportación (de 12 atados de libra) más con el empleo de la alta densidad, por lo que podría llegar a tener un impacto económico significativo. 


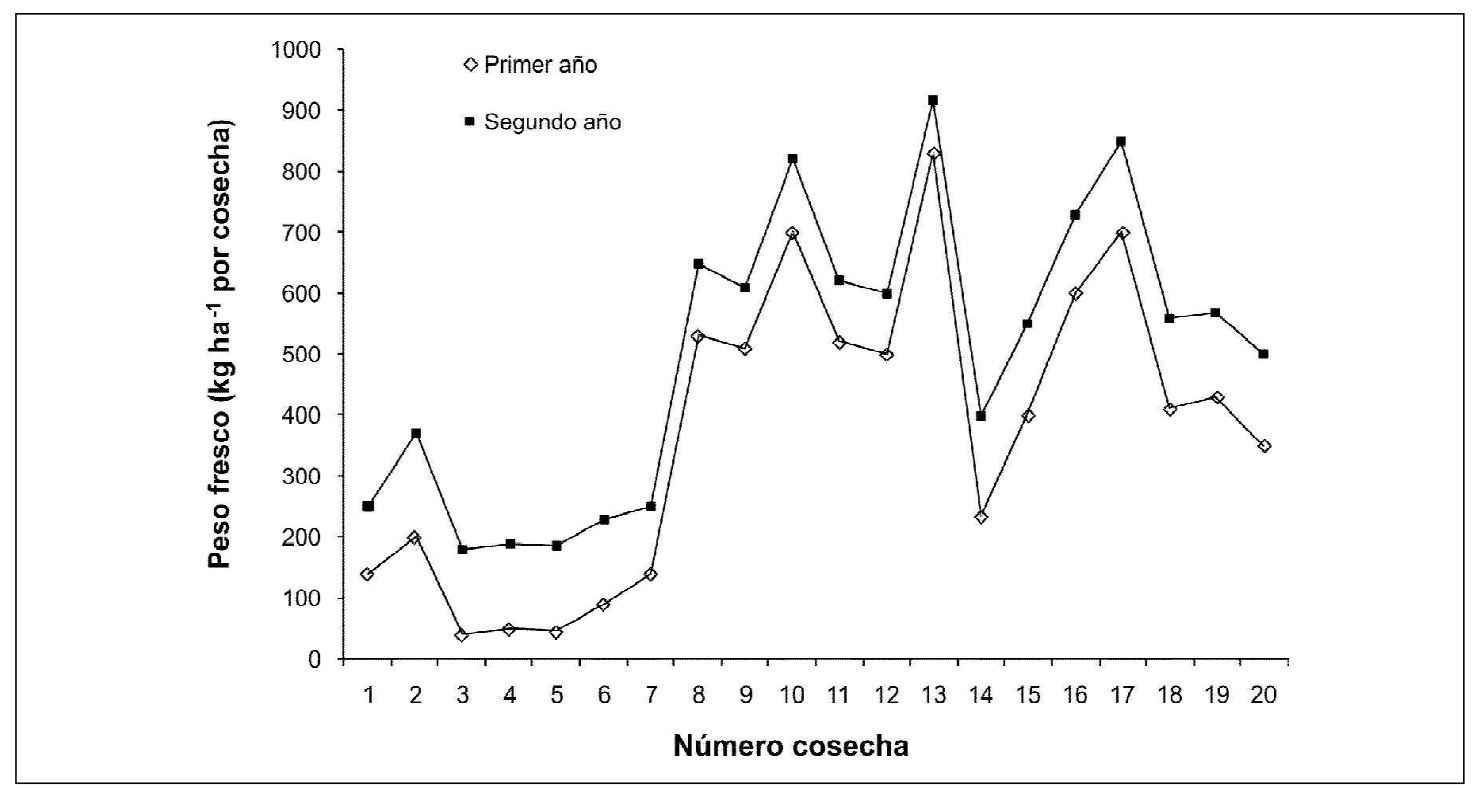

Figura 1. Evolución del peso fresco comercial de una plantación adulta de espárrago verde a lo largo del bienio estudiado.

En la figura 2 se observa la evolución productiva promedio a lo largo del período de cosecha para las dos densidades consideradas; Ios picos se deben a las condiciones climáticas que favorecieron en determinados momentos todas las variables en estudio.

Se observaron diferencias significativas favorables en el peso fresco obtenido a partir de coronas grandes y medianas, en comparación con aquellas de tamaño chico, debido a que existe una diferencia de $2 \mathrm{t}$ entre coronas grandes y chicas, y 1,4 t entre medianas y chicas (tabla 1); estos valores se traducen en una diferencia de 333 y 233 cajas de exportación, respectivamente, para los dos tamaños mayores, respecto de coronas chicas. Esta diferencia en el peso fresco obtenido se debe, posiblemente, a que las plantas solo han mostrado gran efecto de competencia intraespecífica en los dos tamaños superiores, ya que, en promedio, por planta se logró un rendimiento superior en las dos mayores, pero estadísticamente similar (para coronas grandes, $0,450 \mathrm{~kg}$, y para medianas, 0,421 kg), mientras que en las de tamaño inferior fue de 0,354 kg por planta.

El marco de plantación utilizado, de 0,25 y 0,35 $\mathrm{m}$ entre plantas, representó un límite para el crecimiento espacial de las coronas, pues una vez se aproximaron entre sí, ya no pudieron continuar con su ritmo exponencial de crecimiento, lo cual motivó que tanto las coronas medianas como las grandes produjeran estadísticamente el mismo rendimiento. El número de turiones no se habría afectado aún por dichas limitaciones, por lo que las plantas provenientes de las AG siguen produciendo un mayor número proporcional de turiones respecto de los otros dos tamaños, como oportunamente se indica en el ítem correspondiente.

La actividad fotosintética sobreviene principalmente en los cladófilos, durante el período estival. Los fotoasimilados más importantes, acumulados en las raíces de reserva, son carbohidratos, cuya concentración en peso varía de 9,5 a 19,4\% en función de la variedad (Falavigna, 2003). La concentración de carbohidratos en las raíces, lograda por el cultivo durante el período vegetativo estival anterior, expresada en porcentaje de azúcares reducidos y no reducidos, ha sido documentada por Robb (1984). Durante la primavera, cuando la emisión de turiones inicia y la temperatura del terreno se mantiene sobre $12^{\circ} \mathrm{C}$ durante al menos 7 días, la concentración de carbohidratos disminuye progresivamente, ya sea en número de turiones como en las nuevas raíces y yemas, que se desarrollan exclusivamente absorbiendo agua del terreno y utilizando las sustancias de reservas de las raíces del año precedente. Por tal motivo, 


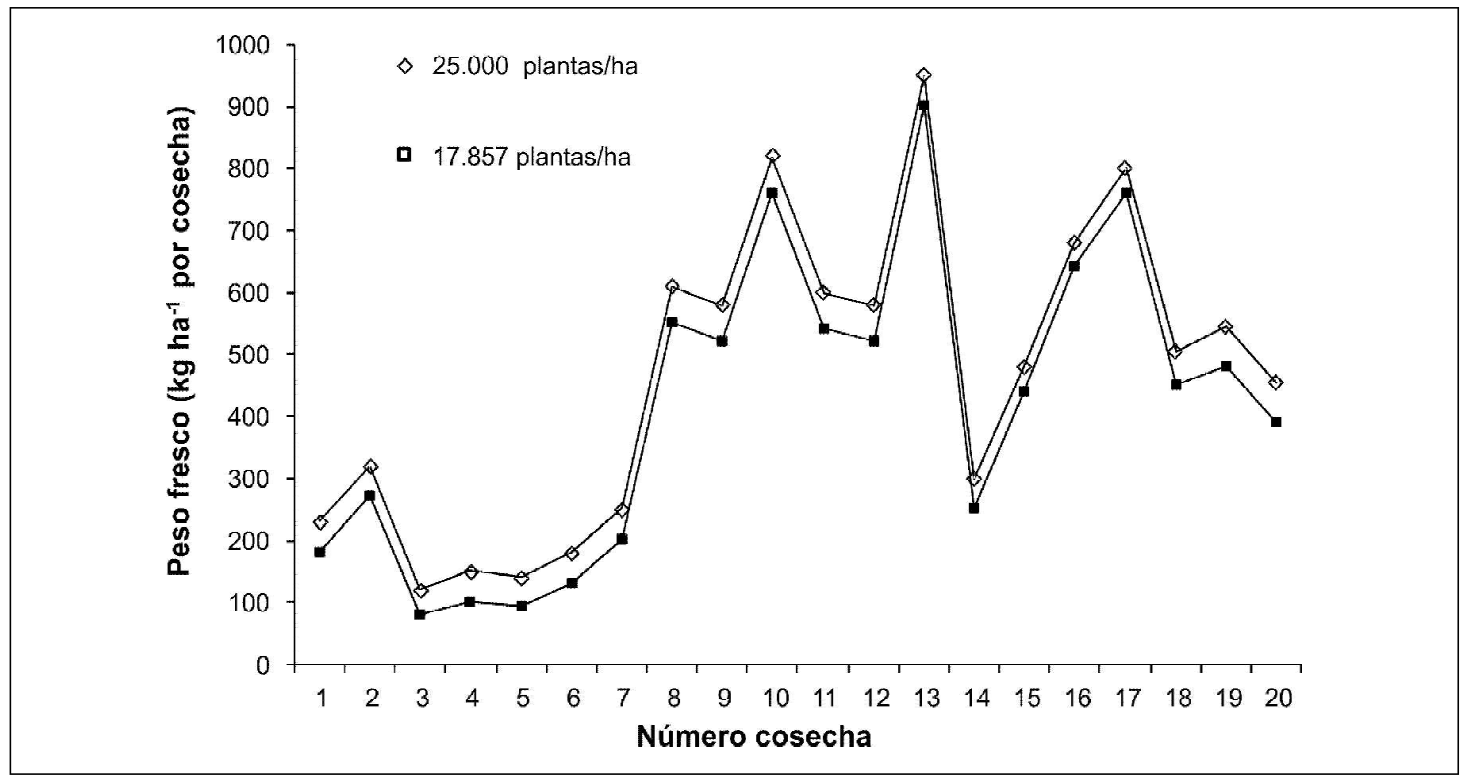

Figura 2. Evolución del peso fresco comercial según densidad de plantación a lo largo del bienio estudiado.

la productividad no depende del manejo inmediatamente anterior a la cosecha, sino de las condiciones del cultivo en cuanto al período vegetativo anterior y de las limitaciones de diferente índole que pudiera haber tenido, como las espaciales para su crecimiento lateral, ya sea por la densidad o por el tamaño adquirido previamente.

El empleo de coronas grandes y medianas, con yemas más desarrolladas respecto de las chicas, desde el comienzo de la vida productiva de la plantación ha incidido favorablemente, ya que han continuado produciendo turiones de mayor calibre; esto indicaría la necesidad de efectuar plantaciones de coronas grandes producidas a partir de almácigos de baja densidad, y, además, de clasificar adecuadamente las coronas que se van a utilizar, brindando a cada una un manejo acorde.

\section{Número de turiones}

En el período estudiado se encontraron diferencias inversas en el rendimiento, ya que en el primer año estudiado el número de turiones fue mayor que en el segundo. También se encontraron diferencias en los tamaños de coronas utilizados y no así en las densidades (tabla 2). No obstante haberse dado un crecimiento en el volumen total producido, no ocurrió lo mismo con el número de turiones logrados, ya que los resultados indican que hubo una diferencia del $11 \%$ a favor del primer año (tabla 2). Esto indicaría que las plantas ya habrían entrado en competencia en el octavo año desde el inicio del cultivo. Posiblemente estas diferencias se deban a que las $A G$ siguen teniendo yemas de mayores dimensiones, pero en menor número, debido a que habrían llegado al límite de su expansión; por tal motivo, el peso fresco fue superior durante el segundo año.

En el caso del espárrago, la cantidad de fotoasimilados almacenados en las raíces actuaría como recurso limitante en la determinación del número y el diámetro de los turiones, de modo que un mayor número implica un menor diámetro y viceversa, existiendo una compensación entre ellos (Cointry et ál., 2000). Los resultados obtenidos en este estudio se corresponden con los encontrados por Ellison (1986), quien Ilevó a cabo un trabajo con las mismas plantas durante tres años consecutivos, en donde observó que los rendimientos máximos provinieron de plantas que tenían muchos turiones grandes, y que las plantas con muchos turiones pequeños o con pocos turiones grandes no tuvieron altos rendimientos.

En el primer año se obtuvieron 18,60 turiones/ planta, mientras que en el segundo, 16,54 


\begin{tabular}{|l|c|c|c|c|}
\hline \multicolumn{4}{|l|}{ Tabla 2. Número y peso de turiones cosechados según año, densidad de plantación y tamaño de corona. } \\
\hline \multirow{2}{*}{ Variable } & & No. turiones/ cosecha & No. turiones/ ha & Peso/turión (g) \\
\hline \multirow{2}{*}{ Año del bienio en estudio } & 1 & $19.927 \mathrm{a}$ & $398.541 \mathrm{a}$ & $19,00 \mathrm{a}$ \\
\cline { 2 - 5 } & 2 & $19.694 \mathrm{~b}$ & $354.493 \mathrm{~b}$ & $23,00 \mathrm{~b}$ \\
\hline \multirow{2}{*}{ Densidad } & $25.000 \mathrm{pl} . / \mathrm{ha}$ & $19.773 \mathrm{a}$ & $375.691 \mathrm{a}$ & $22,36 \mathrm{a}$ \\
\cline { 2 - 5 } & $17.857 \mathrm{pl} . / \mathrm{ha}$ & $19.860 \mathrm{a}$ & $377.335 \mathrm{a}$ & $19,49 \mathrm{a}$ \\
\hline \multirow{3}{*}{ Tamaño de corona } & Chico & $16.874 \mathrm{c}$ & $320.606 \mathrm{c}$ & $23,64 \mathrm{a}$ \\
\cline { 2 - 5 } & Mediano & $19.856 \mathrm{~b}$ & $377.264 \mathrm{~b}$ & $23,91 \mathrm{a}$ \\
\cline { 2 - 5 } & Grande & $22.731 \mathrm{a}$ & $431.889 \mathrm{a}$ & $22,32 \mathrm{a}$ \\
\hline
\end{tabular}

Los promedios en cada variable con letras distintas indican diferencia significativa según la prueba de $\operatorname{LSD}(P<0,05)$.

turiones/planta. En promedio, de los dos años, se destacó la cosecha 13, en la que se cosecharon 33.403 turiones, seguida de las cosechas 10 y 17 , con alrededor de 33.000 turiones. En las cosechas 3, 4 y 5 se cosecharon menos turiones, en promedio se obtuvieron 7.000 turiones (tabla 2). En cuanto al número de turiones, se logró en promedio 17,57 turiones por planta, sin presentarse diferencias significativas entre las dos densidades evaluadas.

Se encontró una relación directamente proporcional entre el número de turiones y el tamaño de corona utilizado; el promedio fue de 37.6587 turiones por ha, con un $13 \%$ más en las coronas grandes y un 15\% por debajo del promedio en las chicas.

\section{Peso por turión}

En el caso del peso por turión no se encontraron diferencias significativas tanto para densidad como para tamaño de corona (tabla 2). En todos los casos se comprobó que la técnica de corona permitió lograr turiones de elevado calibre respecto de diferentes técnicas para el inicio de este cultivo. El mayor peso se obtuvo en la cosecha 10, con $25 \mathrm{~g}$, y el menor, en la cosecha 6 , con $13 \mathrm{~g}$, como era de esperar de acuerdo con la evolución de las condiciones agroclimáticas del año. Durante los dos años en estudio, se obtuvo una diferencia significativa en el peso por turión: en el segundo año se logró $17,4 \%$ más que en el primero (tabla 2); esto posiblemente se deba al menor número de turiones cosechados en ese segundo año, lo que redunda en su mayor peso, dado que se obtuvo un mayor rendimiento en dicho año. Ambas densidades permitieron lograr un rendimiento promedio de $21 \mathrm{~g}$ por turión. El tamaño de corona no influyó significativamente en el peso por turión; en promedio, los tres dieron alrededor de $23 \mathrm{~g}$ (tabla 2).

\section{Proporción de peso según calibres}

Respecto a la proporción de peso cosechado de las tres categorías de calibres considerados, se obtuvieron diferencias significativas para cada una de ellas (tabla 3), en correspondencia con el mayor volumen total obtenido en el segundo año.

A la productividad total lograda con la plantación adulta en estudio es posible asignarle diferentes destinos de acuerdo con las siguientes proporciones obtenidas: $34 \%$ podría destinarse a la Unión Europea, dada su demanda de mayores calibres; $49 \%$, a USA; mientras que el $17 \%$ restante debería tener como único destino el mercado interno y, en lo posible, regional, debido a su mal comportamiento en poscosecha, según los resultados logrados en la segunda parte de la tesis.

En el caso de la UE, además de los $2.814 \mathrm{~kg}$ de los mayores calibres, se podrían admitir (aunque posiblemente a menor precio) los $4.095 \mathrm{~kg}$ logrados de calibre intermedio, con lo que significaría la posibilidad de exportar casi $7 \mathrm{t} \mathrm{ha}^{-1}$ anuales a dicho mercado.

Por el contrario, a USA, dada su preferencia por calibres intermedios y chicos, existiría la posibilidad de exportar 4 t ha $^{-1}$, en promedio. De sumarle los calibres menores, existe el riesgo de reclamos por su mal comportamiento, lo que afectaría el interés de mantener el 
Tabla 3. Rendimiento de turiones por cosecha obtenido para cada tamaño de turión en cada año.

\begin{tabular}{|l|c|c|c|}
\hline Tamaño de turión & Año & $\mathrm{kg} /$ cosecha & $\mathrm{kg} \mathrm{ha}^{-1}$ \\
\hline \multirow{2}{*}{ Proporción de grandes } & 1 & $126 \mathrm{~b}$ & $2531,09 \mathrm{~b}$ \\
\cline { 2 - 4 } & 2 & $172 \mathrm{a}$ & $3096,21 \mathrm{a}$ \\
\hline \multirow{2}{*}{ Proporción de medianos } & 1 & $184 \mathrm{~b}$ & $3684,32 \mathrm{~b}$ \\
\cline { 2 - 4 } & 2 & $250 \mathrm{a}$ & $4506,91 \mathrm{a}$ \\
\hline \multirow{2}{*}{ Proporción de chicos } & 1 & $63 \mathrm{~b}$ & $1273,04 \mathrm{~b}$ \\
\cline { 2 - 4 } & 2 & $86 \mathrm{a}$ & $1557,27 \mathrm{a}$ \\
\hline
\end{tabular}

Los promedios en cada variable con letras distintas indican diferencia significativa según la prueba de LSD $(P<0,05)$.

posicionamiento en dicho mercado, una vez logrado.

Los $1.415 \mathrm{~kg}$ de calibres menores: small y asparagina, admitirían como destinos posibles el mercado interno y, dentro de él, las industrias de II gama (conservas y cremas) y III gama (congelado rápido).

El mayor rendimiento se logró en el segundo año estudiado y correspondió a los turiones de tamaño mediano, mientras que la menor cantidad de peso cosechado por cosecha correspondió al primer año, para los turiones de tamaño chico, como se observa en la tabla 3.

Al analizar los $\mathrm{kg} \mathrm{ha}^{-1}$ cosechados de turiones para los distintos calibres (de elevado calibre [extra large y large], de mediano [médium] y de pequeños calibres [small y asparagina]), se detectaron diferencias estadísticamente significativas para todos los efectos considerados (tabla 4).

El mayor peso obtenido por cosecha correspondió a los turiones de tamaño mediano provenientes de coronas implantadas con alta densidad; mientras que el menor correspondió a los turiones de tamaño chico provenientes de coronas implantadas con baja densidad (tabla 4).

\section{Influencia de la longitud de los turiones de espárrago verde en su comportamiento poscosecha}

En la búsqueda de alternativas de valorización para la producción obtenida de la plantación adulta tradicional en estudio, se pudo comprobar que cortando los turiones a una menor longitud, en correspondencia con las nuevas tendencias en la demanda internacional de este producto, se favorece el comportamiento en poscosecha, extendiendo su vida útil; esto permitiría acceder con menos riesgo de deterioro de la calidad a otros mercados, en comparación con la presentación tradicional en cajas piramidales de 12 atados de $22 \mathrm{~cm}$.

Para las dos longitudes estudiadas, $22 \mathrm{~cm}$ y 12 $\mathrm{cm}$, se produjeron diferencias estadísticamente significativas en el peso promedio de los turiones en evaluación: en el caso de $22 \mathrm{~cm}$, los valores obtenidos fueron: 22,76 g para large; $13,12 \mathrm{~g}$ para mediano, y 6,84 g para small; y en el largo de 12 cm fueron: $14,06 \mathrm{~g}$, large; $8,98 \mathrm{~g}$, mediano y 4,52 $g$, small. Se presentaron diferencias significativas $(P \geq 0,05)$ entre cada uno de los tamaños.

Para ambos largos estudiados la media lograda de las distintas repeticiones resultó estadísticamente superior cuando se envasaron para su etapa de poscosecha ( 1 mes); en el caso de $12 \mathrm{~cm}$ fue de $10,26 \mathrm{~g}$ envasados y $8,12 \mathrm{~g} \sin$ envasar; mientras que a $22 \mathrm{~cm}$ fue de $15,73 \mathrm{~g}$ envasados y $12,76 \mathrm{~g}$ sin envasar, registrándose diferencias significativas $(P \geq 0,05)$ entre los dos tipos de envasado. Estos resultados correspondieron a una diferencia de 5,47 g para los envasados y de 4,64 g para los no envasados, a favor de los largos.

\section{Evolución de la pérdida de peso fresco para diferentes longitudes de turiones en tres calibres}

En general, los turiones cortos $(12 \mathrm{~cm}$ ) mantuvieron mayor pérdida de peso fresco, en

\begin{tabular}{|c|c|c|c|}
\hline Tamaño de turión & $\begin{array}{c}\text { Densidad } \\
\text { (plantas/ha) }\end{array}$ & $\mathrm{kg} / \mathrm{cosech}$ & $\mathrm{kg} \mathrm{ha}^{-1}$ \\
\hline \multirow{2}{*}{ Proporción de grandes } & 25.000 & $157 \mathrm{a}$ & $2.983 \mathrm{a}$ \\
\hline & 17.857 & $139 \mathrm{~b}$ & $2.641 \mathrm{~b}$ \\
\hline \multirow{2}{*}{ Proporción de medianos } & 25.000 & 228 a & $4.332 \mathrm{a}$ \\
\hline & 17.857 & $202 b$ & $3.838 \mathrm{~b}$ \\
\hline \multirow{2}{*}{ Proporción de chicos } & 25.000 & $79 a$ & $1.501 \mathrm{a}$ \\
\hline & 17.857 & $69 \mathrm{~b}$ & $1.311 b$ \\
\hline
\end{tabular}

Los promedios en cada variable con letras distintas indican diferencia significativa según la prueba de LSD $(P<0,05)$ 
comparación con los turiones largos (tabla 5). Para los turiones cortados a $22 \mathrm{~cm}$, la evolución del peso fresco a lo largo del período de poscosecha dependió en mayor medida del calibre, respecto de los cortos.

En los casos de los calibres médium y small se produjeron diferencias de $6 \%$ en el peso fresco, mientras que en large resultó inferior a 1\%; posiblemente esto se deba a las diferencias considerables en el peso para una superficie expuesta determinada según se trate de cortos o largos. Para el calibre large, los turiones cortos y largos tuvieron un comportamiento similar (tabla 5).

\section{Proporción de turiones que mantuvieron sus características organolépticas}

En la necesidad de profundizar el estudio del comportamiento de la nueva modalidad de presentación de los turiones cortos, se efectuó un análisis de la evolución del peso fresco no solo de los turiones cortados con la menor longitud, sino también de largos, presentados en ambos casos envasados y sin envasar.

Se observó la existencia de una relación directa entre el calibre y el largo respecto de la proporción de turiones que mantuvieron sus características (tabla 5); a mayor calibre fue mayor la proporción, cuando los turiones fueron conservados envasados en condiciones adecuadas. En la presentación tradicional, el deterioro fue máximo para todos los calibres a lo largo del mes del período de estudio En el caso de los cortos se pudo encontrar que si están envasados el $81 \%$ de los turiones conservaron sus propiedades, mientras que si no lo están es de $0 \%$. En los turiones largos se observó que el 31\% mantuvieron sus características organolépticas; esto demuestra la conveniencia no solo de utilizar Iongitudes más cortas, demandadas por algunos mercados, sino de acompañarlas con un tipo de envasado acorde, que además de resaltar sus características contribuya a mejorar su comportamiento, brindando al producto un mayor valor agregado y optimizando su calidad.

\section{Evaluación del envasado de turiones de diferentes longitudes}

Al finalizar el ensayo, todos los turiones no envasados, tanto cortos como largos, presentaban parámetros organolépticos no
Tabla 5. Influencia de la longitud y de los calibres de los turiones en la pérdida de peso fresco y en el mantenimiento de sus características organolépticas durante la poscosecha.

\begin{tabular}{|l|c|c|c|c|}
\hline \multirow{2}{*}{ Calibre } & \multicolumn{2}{|c|}{ Pérdida de peso (\%) } & \multicolumn{2}{c|}{$\begin{array}{c}\text { Mantenimiento } \\
\text { característica (\%) }\end{array}$} \\
\hline Small & Cortos & Largos & Cortos & Largos \\
\hline Medium & 71,42 & 63,63 & 57,14 & 14,28 \\
\hline Large & 69,23 & 65,00 & 85,71 & 28,57 \\
\hline
\end{tabular}

comerciales. El largo de los turiones incidió significativamente en el período de conservación, resultando conveniente en un 3,6\% los cortos sobre los largos. En el calibre small, el deterioro resultó exponencial y muy superior a los calibres médium y large; por lo que los empresarios deben tener presente que el destino de dichos turiones debiera ser mercados cercanos y brindarles prioridad en la etapa de comercialización.

No obstante la conveniencia encontrada en la optimización de la calidad en el comportamiento en poscosecha de turiones cortos, es importante considerar que en caso de utilizar dicho largo sería necesario intensificar la frecuencia de cosecha, a fin de propiciar la optimización del rendimiento total comercial, que de otro modo disminuiría de manera notable. En dicho caso surge la necesidad de cosechar turiones de un mínimo de $14 \mathrm{~cm}$.

\section{CONCLUSIONES}

- La productividad del esparrago al inicio de la etapa adulta mantiene una tendencia creciente, y se pueden lograr valores superiores a 8 t ha $^{-1}$.

- De las dos densidades estudiadas, la de 25.000 plantas ha-1 permite lograr rendimientos superiores en turiones.

- Existe una relación directa entre el tamaño de corona y la productividad, por lo que es conveniente utilizar plantines superiores a $100 \mathrm{~g}$.

- El sistema tradicional de coronas en la etapa adulta del cultivo permitiría exportar casi la totalidad de la producción a mercados 
distantes, como la Unión Europea y Estados Unidos, lo que podría significar un alto beneficio económico para el productor, dado el elevado precio de este producto en dichos mercados.

- Debido a la reciente incorporación de turiones cortos al mercado, y a su creciente demanda, los productores deberían tener en cuenta esta gran oportunidad para comercializar un producto innovador, ya que, además, cuenta con la ventaja de tener mayor vida útil, debido a su menor deshidratación en poscosecha. A su vez, a mayor calibre existen mayores posibilidades de conservación, por lo cual, para este fin, se puede usar la proporción de la producción correspondiente a los calibres medium, mientras large sería la más adecuada cuando el destino es la exportación a mercados distantes.

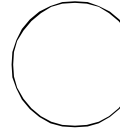

Asprelli, P.D.; F.S. López-anido y E.L. Cointry. 2005. Caracteres agronómicos en cultivares de espárrago de diferentes edades y manejos. Pesqui. Agropecu. Bras. 40(1), 47-52.

Benajes-Sanahuja, S. 1990. El espárrago. Ediciones Mundi-Prensa, Madrid.

Castagnino, A. 2006. Comportamiento del cultivo de espárrago verde a diferentes densidades iniciado mediante el sistema tradicional de arañas. Agronomía Trop. 56(1), 121-127.

Cointry, E.; F.S. López Anido; I. Gatti; V.P. Cravero; I.T. Firpo y S.M. García. 2000. Early selection of elite plants in asparagus. Bragantia 59(1), 21-26.

Ellison, J.H. 1986. Asparagus breeding. pp. 521-569. En: Basset, M.J. (ed.). Breeding vegetables crops. Westport, CT.

Falavigna, A. 2006. I Punti critici dee'asparago in campo en el post-raccolta. La stessa tecnica per venti secoli: la fossa per le «zampe». L'Informatore Agrario 52-56.

Falavigna, A. 2004. Strategia per la ottimizazione e valorizzazione de la produzione di asparago in Sicilia. Editorial Grillo e Famá, Spadafora, Italia. pp. $39-40$

Falavigna, A. 2003. Le conoscenze tecnico-scientifiche e l'attività di ricerca condotta nel PROM al servizio del produttore agricolo. Progetto di Ricerca per potenziare la competitività di Orticole in aree Meridionali. Delibere CIPE 17/2003 e 83/ 2003. pp. 1-77.

Falavigna, A. y A.D. Palumbo. 2001. La coltura dell'asparago.Editorial Calderini Edagricole, Bologna, I talia.

\section{REFERENCIAS BIBLIOGRÁFICAS}

Instituto Interamericano de Cooperación para la Agricultura (IICA) 2007. Guía práctica para la exportación a EE.UU. Nicaragua. En: http:// www.iica.int.ni; consulta: noviembre de 2009

Laemers, H.M.A. 2008. Asparagus developments in The Netherlands, Europe and Wordwide. $5^{\mathrm{a}}$ Forum Europeo dell' Asparago. Acta Euroasper 2008.

López-Gálvez, G. y M. Cantwell. 1996. Los productos de cuarta gama en Estados Unidos. Horticultura $117,33-38$

Manrique, K.K. 1998. Nociones del manejo de postcosecha. Depto. de Mejoramiento y Recursos Genéticos. En: http://wWw.cipotato.org/ newindex.asp; consulta: noviembre de 2009.

Montanari, V. 1996. I mezzi tecnici per la coltura in vivaio. Informatore Agrario 52(29 Suppl.), 25-35.

Nichols, M.A. y D. Woolley. 1985. Growth studies with asparagus. pp. 287-297. En: Proc. Int. Asparagus Symp. University of Guelph, Ontario, Canada.

Rivera, I. y J. Rodríguez. 1999. Perfil de mercado: espárrago. Documento de trabajo No. 5. En www.inta.gov.ar/ies; consulta: noviembre de 2009.

Robb A.R. 1984. Physiology of asparagus (Asparagus officinalis) as related to production of the crop. New Zeal. J. Exp. Agr. 12, 251-260.

Romojaro, F.; F. Riquelme; T. Pretel; G. Martínez; M. Serrano; C. Martínez; P. Lozano; P. Segura y P. Luna. 1996. Nuevas tecnologías de conservación de frutas y hortalizas. Ediciones Mundi-Prensa Madrid. pp. 41-58.

Secretaría de Agricultura, Ganadería Pesca y Alimentos (SAGPyA). 2007. Protocolo de calidad de espárrago fresco. Buenos Aires. 